\title{
Word Reading Fluency as a Serial Naming Task
}

\author{
Athanassios Protopapas \\ University of Oslo and National and Kapodistrian University of Athens \\ Katerina Katopodi \\ National and Kapodistrian University of Athens \\ Angeliki Altani and George K. Georgiou \\ University of Alberta
}

In press, Scientific Studies of Reading. doi: 10.1080/10888438.2018.1430804

\section{Author Note}

Athanassios Protopapas, Department of Special Needs Education, University of Oslo, Norway, and Department of Philosophy \& History of Science, National and Kapodistrian University of Athens, Greece. Katerina Katopodi, Graduate Program in Basic and Applied Cognitive Science, National and Kapodistrian University of Athens, Greece. Angeliki Altani and George K. Georgiou, Department of Educational Psychology, University of Alberta, Canada.

Correspondence concerning this article should be addressed to Athanassios Protopapas, PO Box 1140, Blindern, 0318 Oslo, Norway; Phone: +47 228 57705; e-mail: athanasios.protopapas@isp.uio.no 


\begin{abstract}
Word list reading fluency is theoretically expected to depend on single word reading speed. Yet the correlation between the two diminishes with increasing fluency, while fluency remains strongly correlated to serial digit naming. We hypothesized that multielement sequence processing is an important component of fluency. We used confirmatory factor analyses with serial and discrete naming tasks with matched items, including digits, dice, objects, number words, and words, performed by about one hundred Greek children in each of Grades 1, 3, and 5. Separable serial and discrete factors emerged across grades, consistent with distinct skill dimensions. Loadings were greater for serial than discrete, suggesting that discrete processing does not fully determine serial processing. Average serial performance differed more than discrete between grades, consistent with improvement beyond single-item speed. Serial word reading aligned increasingly with the serial factor at higher grades. Thus, word reading fluency is gradually dominated by skill in simultaneously processing multiple successive items through different stages (termed "cascading"), beyond automatization of individual words.
\end{abstract}

Keywords: fluency, reading, RAN, serial naming, word recognition 


\section{Word Reading Fluency as a Serial Naming Task}

Reading fluency, that is, "the ability to read a text quickly, accurately, and with proper expression" (National Reading Panel, 2000, p. 3-5) has long been recognized as a "critical component of skilled reading" (p. 3-1), a "key construct" (Rasinski, Reutzel, Chard, \& Linan-Thompson, 2012, p. 286) involving "every process and subskill involved in reading" (Wolf \& Katzir-Cohen, 2001, p. 220). It has become clear that reading accuracy is not sufficient for functional literacy and that accuracy gains do not transfer to fluency (Torgesen, 2005). However, despite advances in assessment (Cummings \& Petscher, 2016), theoretical developments have lagged behind. For all the progress in documenting the remarkable stability of individual differences in reading fluency through elementary education and beyond (Georgiou, Papadopoulos, \& Kaizer, 2014; Landerl \& Wimmer, 2008), the construct itself remains vague and there is little understanding of the cognitive mechanisms underlying fluent reading.

From the earlier attempts to understand the facility and fluidity of skilled reading using the concepts of automatization (LaBerge \& Samuels, 1974) and "rauding" (Carver, 1997) through the more recent proposals (e.g., Fuchs, Fuchs, Hosp, \& Jenkins, 2001; Hudson, Pullen, Lane, \& Torgesen, 2009; Hudson, Torgesen, Lane, \& Turner, 2012; Kuhn, Schwanenflugel, \& Meisinger, 2010; Kuhn \& Stahl, 2003; Rasinski et al., 2012; Rasinski, Rikli, \& Johnston, 2009; Wolf \& Katzir-Cohen, 2001), fluency has been thought to comprise two main aspects: A word-level aspect, concerning identification of words and access to their associated lexical contents; and a higher-level aspect, concerning linguistic processing of sentences and texts by oral language skills, involving expressive prosody and understanding of text meaning. At the word level, researchers have focused predominantly on the efficiency of processing single words, as if they were isolated and independent from one another. Fast and effortless recognition of individual 
words, termed "sight word reading" (Ehri, 2005, 2014), is tacitly considered to be the necessary and sufficient condition at the word level for achieving reading fluency. If this were the case, then-in the absence of sentence-syntactic and text-semantic requirements-reading fluency should be strongly predictable by individual word recognition efficiency.

However, reading fluency is typically measured with multi-word tasks, either lists of unrelated words (or pseudowords) or continuous meaningful texts. Word list reading fluency (often termed "efficiency") is commonly defined as a metric combining accuracy and speed of reading aloud a list of words simultaneously displayed (e.g., in columns; Torgesen, Wagner, \& Rashotte, 1999). It seems intuitively clear that the sequential nature of dealing with multiple words-whether arranged in rows or in columns, and whether forming meaningful sentences or not-is an inextricable aspect of fluent reading. Yet this is nowhere to be seen in theoretical treatments of fluency, which typically refer only to the efficiency of processing individual words and to supralexical effects concerning phrasal grammatical structure and meaning.

Although the importance of efficient individual word recognition is undeniable, it cannot be the only critical factor underlying fluent reading, because the speed of individual word reading predicts word list reading only moderately, and this correlation weakens substantially with increasing skill (de Jong, 2011; Protopapas, Altani, \& Georgiou, 2013). Thus, the sequential processing of a series of items that are simultaneously available and must be dealt with via an endogenously controlled process (i.e., governed by the reader's own planning) has been highlighted as a potential “missing link" to understanding fluency (Altani, Protopapas, \& Georgiou, 2017b; Protopapas et al., 2013; Zoccolotti, De Luca, \& Spinelli, 2015). The link is provided by serial naming tasks, commonly termed "rapid automatized naming" (RAN). RAN 
consists in a self-generated sequential naming procedure going through a series of repeated familiar visual stimuli (e.g., letters, digits, colors, objects), thus minimizing efforts concerning identification and articulation (Wolf \& Bowers, 1999; Wolf, Bowers, \& Biddle, 2000). Importantly, serial digit naming predicts serial word reading (i.e., word list fluency) beyond the contribution of discrete words (i.e., individual word reading), equally across a wide spectrum of orthographies (Altani et al., 2017a).

Protopapas et al. (2013) claimed that serial word reading starts off similar to discrete word reading, in that beginner readers deal with words one at a time and move on to the next word in a sequence only after they have completed processing the previous word. This strict sequencing results in a strong correlation between discrete and serial word reading for Greek children attending Grade 2 (and beginner "Class 1" Dutch readers; de Jong, 2011). A very different picture has emerged for Grade 6 children, where serial word reading aligned more closely with serial digit naming than with discrete word reading (similar to intermediate "Class 2" Dutch readers; de Jong, 2011). This suggests that, once words are processed more efficiently, the endogenous procedure of managing multiple stimuli in a sequence becomes a dominant factor in word list reading, beyond the efficiency of processing individual words. The crucial aspect of this procedure was hypothesized to concern simultaneous processing of adjacent items at different stages: One word is processed while the previous one is uttered, the next one is viewed, and one further down is previewed, resulting in an effectively parallel processing pipeline termed "cascaded" processing.

This hypothesis refers to word-level processing, observable with lists of unrelated words. Thus, here we refer to multi-word but not supra-lexical processing. This kind of multi-word sequencing must also occur in the course of regular reading, that is, with grammatically structured and meaningful sentences making up texts, and 
must partially underlie fluent reading as commonly understood. It stands to reason that reading can only be fluent and expressive if one has planned the expression ahead of time, that is, has processed the upcoming words well before uttering them.

Thus, in addition to the supra-lexical element concerning grammatical and semantic processing, which must form part of fluent text reading, we propose that word-level multi-element processing is a distinct, necessary component as well; and that this component is captured not only by word list reading but also by rapid naming tasks. This speculative idea, indirectly supported by eye-movement research in both reading (de Luca et al., 2013; Laubrock \& Kliegl, 2015; Marx, Hutzler, Schuster, \& Hawelka, 2016) and digit naming (Gordon \& Hoedemaker, 2016; Pan et al., 2013; see also Al Dahhan, Kirby, \& Munoz, 2016), can account for the well-known observations that upcoming words are parafoveally previewed and, more importantly, the eye looks ahead of the spoken word when reading aloud, creating an "eye-voice span", which allows skilled readers to comprehend a sentence before having to pronounce it, and therefore to read it with appropriate expression, resulting in the hallmark prosody of fluent reading. These observations are hardly new (e.g., Buswell, 1921); the novel claim made here is just that the ability to efficiently form and maintain processing cascades is a distinct factor in fluent reading that can be indexed by serial rapid naming.

If this idea is on the right track then we should be able to observe the emergence of a serial processing construct, indexed primarily by digit naming, gradually encompassing word list reading in more advanced readers. Protopapas et al. (2013) claimed such a developmental alignment with a set of tasks (words, digits, and objects) that was too small to disentangle the effects of cascaded processing from specific task features. Therefore, in the present study we examined the common variance shared among a larger number of serial naming tasks indexing a "serial" latent construct. This 
is compared to a "discrete" latent construct capturing variance shared among corresponding discrete naming tasks, to account for task demands such as item recognition and articulation. In a cross-sectional approach, we tracked this common variance from beginner (Grade 1) through intermediate (Grade 3) and relatively advanced (Grade 5) reading skill. We constructed the tasks to be as similar as possible in articulatory and other processing demands, using the same words whenever possible and equating words on several variables when different words were unavoidable. This permits interpretation of nonshared variance free from material confounds.

To expose differences between the serial and discrete naming tasks we have administered all tasks to all children in both serial and discrete naming formats, aiming to test the following hypotheses:

(H1) Serial word reading will increasingly align with serial digit naming (i.e., RAN) in higher grades, consistent with the rising dominance of cascaded processing over word list reading. This implies greater factor loadings in higher grades.

(H2) Individual differences in serial naming will not fully parallel discrete naming, consistent with the distinct dimension of cascaded processing in serial tasks. This implies distinct serial and discrete factors across grades.

(H3) Average performance in serial naming will not track performance in discrete naming but will follow a distinct developmental trajectory. This implies different rates of development between serial and discrete tasks.

The general idea behind these specific predictions is to test whether (a) serial naming requires something beyond discrete naming; and (b) word list reading fluency, as a serial word reading task, diverges from single word reading to increasingly align with serial naming at higher grades (i.e., higher levels of reading skill). 


\section{Method}

\section{Participants}

Participants included 100 children from Grade 1 (53 girls, age $M=82.8$ months, $S D=3.4$, range 77-89), 103 from Grade $3^{1}$ (53 girls, age $M=107.1$ months, $S D=3.5$, range 100-113), and 99 from Grade 5 (54 girls, age $M=130.0$ months, $S D=3.4$, range 124-136). All were native speakers of Greek from a middle socio-economic background, attending regular classrooms in public schools, recruited voluntarily from the general population, and not experiencing intellectual, sensory, or behavioral difficulties. Research permission from the Greek Ministry of Education and parental consent were obtained before testing.

\section{Materials}

Materials were matched across tasks to the extent possible, aiming to minimize differences in lexical access and articulatory planning and the between-task variance that might be absorbed by such differences, thus maximizing variance reflecting task demands rather than item-related properties.

Reading tasks included number words and words. Number word reading included nine repetitions of each of the four Greek words corresponding to the numbers 2, 3, 5, and 6 (/ðio/, /tria/, /pede/, /eksi/, respectively). All four words are bisyllabic, stressed on their first syllable. Word reading included two sets of 36 high-frequency bisyllabic words, matched in frequency, number of graphemes and phonemes, and syllabic structure to each other and to the four number words.

Naming tasks included digits, dice, and objects. Digit naming included nine repetitions of each of the four digits $2,3,5$, and 6 . Dice included nine repetitions of each

\footnotetext{
1 The Grade 3 data have been previously reported in a cross-linguistic study (Altani et al., 2017a).
} 
of the dice faces for the same four numbers. Objects included nine repetitions of each of four drawings selected to be named with words matched in frequency, number of syllables, graphemes, and phonemes, stress pattern, and syllabic structure to the four number words. These were /oplo/ "gun", /bala/ "ball”, /ora/ "time”, and /plio/ "ship."

Frequency matching was done on the basis of both adult text corpora (IPLR; Protopapas, Tzakosta, Chalamandaris, \& Tsiakoulis, 2012) and children's school textbooks for language arts (lists for Grades 1-3 and 1-6), to ensure comparable familiarity to the words for the numbers, objects, and written words. All words and their length and frequency counts are listed in the Appendix.

\section{Procedure}

Serial tasks. All 36 items were presented simultaneously in grid formats of four rows of nine on a $15.4^{\prime \prime}$ computer screen, in a quasi-random order precluding item repetitions (fixed across participants). Children were instructed to name out loud all items (digits, dice, or object) or read all words as quickly as possible. Instructions and practice items were provided prior to each trial to ensure compliance with task demands.

Discrete tasks. Digits or words were presented individually, centered on the screen, in a fixed quasi random order precluding immediate repetitions. Children were instructed to name out loud each item (or read each word) as quickly as possible. Each task was preceded by four practice items, including feedback.

For both serial and discrete tasks, item presentation and response recording was controlled by the DMDX experimental display software (Forster \& Forster, 2003). Items were presented in black 20-pt Consolas font on a white background and remained on the screen until the experimenter pressed a key to proceed to the next item, as soon as complete production of a response was registered. Individual responses were recorded 
in audio files through a head-mounted microphone and the total naming or reading time was determined off-line using CheckVocal (Protopapas, 2007).

For discrete tasks, naming or reading times of individual items were recorded; for serial tasks, total naming or reading times of the entire array were recorded. All recorded response times (RTs) analyzed below included both onset latency and articulation time. Therefore, measures are directly comparable between serial and discrete tasks. RTs were transformed to a common scale of "items per second" by inversion. For discrete tasks, a single score for each participant was computed by averaging RTs across correctly named (or read) items. Errors in serial tasks were ignored, as typically done in serial naming studies. ${ }^{2}$

Testing took place in April to June (8-10 months into the academic year). The naming and reading tasks were administered individually to each child in random order during a 40-minute session including additional tasks not reported here.

\section{Results}

All statistical analyses were carried out in $\mathrm{R}$ version 3.3.2 ( $\mathrm{R}$ development core team, 2016). A small number of individual outlier data points ( 3 in Grade 1 and 2 in Grade 5) were removed by examination of quantile-quantile (Q-Q) plots, on the "items per second" scale, for each task and grade. Descriptive statistics for the cleaned-up dataset are presented in Table 1. (Accuracy information is available in the online supplementary materials, Table S1). Mean performance per task and grade is displayed in Figure 1. (See individual Q-Q plots and bivariate scatterplots in Figures S1-S8). The resulting distributions per task and grade were generally well approximated by the normal distribution (normality tests in Table S2).

\footnotetext{
2 The effect of ignoring errors was negligible; see Table S13 and Figure S12 in the online supplementary materials.
} 


\section{Analysis of Individual Differences}

Table 2 lists intercorrelations among tasks for each grade. We sought to define format-specific factors accounting for shared variance between discrete naming tasks, on the one hand, and serial naming tasks, on the other. Confirmatory factor analyses were conducted separately for each format and grade using R package openMX 2.0 (Neale et al., 2016). Thus, six single-factor models were fit, with latent disturbances fixed to one and other parameters (loadings, residuals, and means) freely estimated. Theoretically plausible residual covariance parameters were added to achieve model fit. The fit of all models was acceptable to excellent (Figure S9). The residual variances of the discrete task models were consistently lower than those of the serial task models (an assertion statistically supported by nonoverlapping confidence intervals), indicating that discrete tasks share more common variance than serial tasks.

To see if loadings change significantly between grades we forced the models to be as similar as possible. Specifically, the three grade-specific models for each factor were combined into a single multigroup unconstrained model. Subsequently, we constrained individual model parameters to be equal across adjacent grades (i.e., between Grades 1 and 3, and between Grades 3 and 5), starting with factor loadings and proceeding with residual errors, and finally residual covariances, when applicable. Equality constraints were retained when the resulting model was not significantly different from the unconstrained one and its chi-square fit statistic remained nonsignificant. The resulting models, shown in Figure 2 (serial with 37 parameters and discrete with 32 parameters; listed in Tables S4 and S6), were not significantly different from the unconstrained models (with 49 and 47 parameters, respectively; listed in Tables S3 and S5). The fit of both models was good. The loadings of number words and words to the discrete naming factor were not significantly different between Grades 3 
and 5, while discrete digits loadings did not differ among any grades. In contrast, the loading of serial words was significantly different between both adjacent grade groups, and serial digits loadings increased slightly between Grades 1 and 3, indicating increasing alignment among these serial tasks (addressing hypothesis H1).

To test whether serial and discrete factors are truly distinct, we followed up with joint multi-group models including both kinds of tasks. The different patterns of residual covariances among grades necessitated additional factors for reading tasks (number words and words) in Grade 1 and for nonsymbolic images (objects and dice) in Grade 5, to achieve acceptable fit. In every case, residual variances from the serial factor exceeded those from the discrete factor, consistent with more uniform processing-hence more homogeneous individual differences-among discrete than among serial tasks. (The joint unconstrained and constrained models are displayed in Figures S10 and S11, with parameter estimates listed in Tables S7 and S8.) Distinct serial and discrete factors were necessary for model fit (addressing H2).

In particular, serial words were decreasingly correlated with discrete words in higher grades, but stably or somewhat increasingly correlated with serial digits (Table 3, augmented with data for Grades 2 and 6 from Protopapas et al., 2013).

\section{Analysis of Average Task Performance}

Thanks to the common metric across all tasks, effects-coded models (Kline, 2016) could be defined to examine factor means and variances (Tables S9 and S10). However, the significantly different loadings between grades (i.e., lack of strong invariance) make them uninterpretable: If different tasks contribute differentially to the construct across grades then latent means are not meaningfully comparable. Thus we examined performance differences across grades for individual tasks.

An omnibus mixed analysis of variance was conducted, with task as within- 
participant factor (5 levels) and grade as between-participant factor (3 levels) using function lme of the nlme package v. 3.1-128 (Pinheiro et al., 2016), followed up by sets of multiple linear contrasts using function glht of package multcomp v. 1.4-6 (Hothorn, Bretz, \& Westfall, 2008). A first set of contrasts examined differences between tasks, separately for each format (serial or discrete) and grade, comparing adjacent tasks in order of descending difficulty (Table S11). There were significant differences among tasks such that words and objects, on the one hand, were more difficult than number words and digits, on the other hand, with dice occupying an intermediate level of difficulty (see Figure 1). A second set of contrasts examined differences among grades for each task in each format (Table S12). For every serial task there were significant differences between first and third grade and between third and fifth grade. However, for discrete tasks, there were no significant differences between third and fifth grade, except for words. That is, there was no significant improvement in discrete naming of digits, number words, dice, or objects between Grades 3 and 5, even though there was significant improvement in serial naming of the same materials.

To examine whether this difference in significance was itself significant, a third set of contrasts examined interactions between format and grade for each task. That is, we compared whether the difference between grades was greater for serial than for discrete tasks, separately for each task and for each grade pair (first vs. third and third vs. fifth). As shown on Table 4, there was a significantly greater difference between grades for serial than for discrete tasks, with only two exceptions, namely dice between Grades 1-3 and objects between Grades 3-5. These were the only two cases in which a between-grades difference in discrete task performance was commensurate with the corresponding between-grades difference in serial task performance. In every other case, serial naming improved more between grades than discrete naming (addressing 
H3). This task asymmetry reached maximum values, both in absolute and in standardized terms (contrast estimates and z scores, respectively, in Table 4), for words and number words between Grades 1 and 3.

\section{Discussion}

The results confirmed that serial and discrete digit naming tasks were strongly aligned with the serial and discrete factor dimensions, respectively, across Grades 1, 3, and 5. This means that digit naming tasks effectively index these dimensions, as hypothesized. Word reading trailed behind other tasks in aligning with the common factors, especially in the serial format, as its loading on the serial factor increased between Grades 3 and 5 (H1). Across grades, two distinct factors (serial and discrete) were necessary for model fit (H2), consistent with separable skill dimensions. Performance comparisons revealed that serial naming rates continued to increase after Grade 3 much more steeply than discrete naming rates (H3), complementing the individual-differences picture with corroborating group-average evidence. In addition, serial naming tasks shared less variance than discrete naming tasks, indicating that they differed more despite the common elements between formats. In other words, serial naming of certain kinds of elements seems to align better with serial digit naming than others. Finally, we found that number word reading behaves like digit naming rather than like word reading, and that object and dice naming were less well aligned with the common factor than other tasks, especially so in the serial format.

These results indicate that there is a dimension of performance applying specifically to serial-format tasks and that the alignment of particular tasks on this dimension is not predictable on the basis of their alignment in the discrete format. In the following we interpret these findings based on the hypotheses that (a) serial naming and reading involve a distinct skill domain, largely indexed by digit RAN, which 
concerns cascaded processing efficiency; and (b) different kinds of elements are differentially amenable to cascaded processing, depending both on their individual processing efficiency (perhaps akin to "automaticity") and their contextual availability. According to this view, word reading fluency cannot be fully explained by recourse to individual word processing efficiency alone but, in addition, requires a complex skill of endogenous sequential processing over and above individual word recognition, and is also influenced by the predictability of words in the contexts in which they appear, as explained below.

\section{Serial vs. Discrete Word Reading}

From the point of view of understanding word list reading fluency, perhaps the most obvious finding that calls for an explanation concerns the correlation between discrete and serial word reading, which diminishes as reading skill increases (Table 3; see also Gasperini et al., 2014; Zoccolotti et al., 2013, 2014). It seems clear that in the first two grades there is a very close association between discrete and serial reading, accounting for almost three quarters of serial (i.e., fluency) variance, suggesting that beginner readers literally read one word at a time. However, from Grade 3 onward, the correlation with discrete word reading drops substantially, to account for well under one quarter of fluency variance by Grade 6 . This is not due to diminished variance or low reliability of discrete word reading because the correlation of discrete word reading with discrete digit naming remains high (cf. van den Boer \& de Jong, 2015; van den Boer, Georgiou, \& de Jong, 2016). In other words, the more fluent one becomes, the less is their fluency attributable to improved individual word reading. In contrast, the correlation of serial word reading to serial digit naming increases somewhat, such that a crossover of importance occurs between Grades 3 and 5, consistent with a higher proportion of serial word reading variance accounted for by serial digit naming than by 
discrete word reading.

Notably, this is not generic "naming speed" variance, because the correlations of serial word reading with discrete digit naming are substantially lower. It is also not generic "processing speed," as typical measures of this construct (visual matching, cross-out) have failed to account for the relationship between RAN and reading (Bowey, McGuigan, \& Ruschena, 2005; Georgiou, Parrila, \& Kirby, 2009) and do not load on a RAN or reading fluency factor (Bowey, Storey, \& Ferguson, 2004; van den Bos, Zijlstra, \& van den Broeck, 2003). Therefore, whatever cognitive processes are involved in speeded naming of individually presented digits cannot be the reason why RAN is increasingly predictive of reading fluency at higher grades. Rather, something beyond individual element naming and beyond individual word recognition seems to take over, as RAN indexes the dominant skill domain once fluency is attained and the importance of individual word reading recedes. This domain does not concern supra-lexical linguistic processes, such as syntactic, semantic, or pragmatic processing, because our measure of fluency was derived with a list of unrelated words, which did not form meaningful sentences and therefore could not have been linguistically engaged in a way that could have facilitated or limited their processing efficiency. In other words, we are talking about a word-level effect, in which higher-level oral language skills (related to grammatical structures and phrasal meanings) can play no appreciable role.

\section{When is Efficient Serial Naming Possible?}

Our results suggest that different materials are differentially amenable to cascaded processing in patterns not predictable from discrete naming performance. This is supported by three findings: First, number words behaved more like digits than like words, especially after Grade 1, both in their average serial and discrete naming times and in their loadings to the serial and discrete factors. Because number words 
were equated to the word lists on relevant psycholinguistic variables, this difference cannot be attributed to superficial features. Rather, the property of constituting a small closed set may have facilitated their processing to the point of effective automatization. This may have been due to primed phonological activation and articulatory planning, on the one hand, and to facilitated recognition due to elevated prior probability, on the other hand. That is, in the number words task the participant chooses among four possible responses, whereas in the words task the recognition of each element potentially involves one's entire vocabulary. Apparently, increased predictability facilitates individual word processing to permit tighter cascading, suggesting that whatever enables fluent processing is not strictly a property of individual items but also depends on their context. One might even go a step further to speculate that an increase in predictability achieved by familiarity may be related to the effectiveness of repeated reading in fluency interventions (Lee \& Yoon, 2017).

The second finding regarding amenability to cascaded processing concerns object and dice naming. These exhibited relatively lower loadings, especially on the serial factor, trailing behind as other tasks were taking off by Grade 5, and requiring a distinct factor in the joint multigroup analysis (labeled "M" in Figures S10-S11). This can be attributed to semantic mediation insofar as objects and, to a lesser extent, dice, are not fixed visual configurations with direct access to corresponding phonological forms but must first be recognized as instances of a concept (or number) and then phonologically mapped via lemma activation (Liu \& Georgiou, 2017; Poulsen \& Elbro, 2013; cf. Roelofs, 2003, 2006). Presumably, further experience with naming these specific images could lead to access to the direct naming route (a transition possibly already underway for the dice; cf. Marx et al., 2016; Pan et al., 2013). Note that the loadings for these "mediated" tasks were lower in the serial than in the discrete tasks, 
revealing that the effect of mediation is not so much in individual naming but, rather, in efficient resource allocation underlying cascaded processing. Similarly, in a comparison between color and shape naming, significant differences in cross-dimensional interference were found despite little difference in individual naming difficulty, consistent with mediation affecting the efficiency of scheduling multiple cognitive components much more than individual stimulus processing (Protopapas, Markatou, Samaras, \& Piokos, 2017).

The third finding regarding amenability to cascaded processing concerns the focus of our interest, namely, words. In the original automaticity hypothesis, LaBerge and Samuels (1974) made clear that automaticity concerns cognitive efficiency conceptualized as autonomy from attention. Therefore, it cannot be ascertained by measuring speed but requires consideration of attentional allocation. This conception can partially account for the puzzle identified above, namely, that the correlation between serial and discrete word naming diminishes with increased skill, if we concede that discrete word naming speed does not index single word processing automaticity.

Our cascaded processing proposal should thus not be viewed as antagonistic to the automaticity hypothesis but, rather, as a complementary extension, to account for a larger range of findings. It not enough to ascribe the critical longitudinally diminishing association to an automaticity construct that is not captured by discrete word reading speed. One must also account for the stable-if not increasing-association between serial word reading and serial digit naming. We would also like to account (a) for the stable longitudinal associations between early rapid naming performance, early word reading fluency, and late reading fluency (e.g., Georgiou et al., 2014; Landerl \& Wimmer, 2008), (b) for the fact that effective remediation of word reading accuracy difficulties does not transfer to fluency gains (e.g., Torgesen, 2005; nor does increasing the speed of 
reading individual syllables; Heikkilä et al., 2013), and (c) for the common finding that dyslexia in transparent orthographies is primarily associated with fluency deficits in the context of adequate accuracy (Landerl, Wimmer, \& Frith, 1997; Share, 2008).

That is, beyond some property of individual words allowing them to be processed efficiently in sequences, which may be called "automaticity," one must also account for a separable skill related to sequence processing, corresponding to our "serial" factor. One would eventually hope to posit a cognitive mechanism regarding the kind of sequential processing involved, interfacing with the automaticity construct to explain when, why, and how certain elements are dealt with in a sequence much more efficiently than others. This is why we believe it is fruitful to think of word list reading fluency as a cascaded serial naming task.

\section{Measuring Reading Fluency}

Reading fluency has been defined in different ways depending on interests and goals, ranging from "the rate of word decoding" (Breznitz, 2006, p. 235), "the ability to decode and comprehend at the same time" (Samuels, 2006, p. 39), to "accuracy, automaticity, and oral reading prosody, which, taken together, facilitate the reader's construction of meaning" (Kuhn et al., 2010, p. 240), among others. It is important to clarify how our proposal relates to more traditional concerns in the reading literature.

As noted above, we take "word reading fluency" to be a measure of efficiency in reading lists of unrelated words. In particular, we have chosen lists of familiar words not expected to pose high decoding demands. In contrast, typical measures of word reading efficiency employ lists of increasingly difficult words, so that performance is potentially co-determined by accuracy limitations. This issue has received little attention although it is relevant both for the study of fluency itself as well as for the relationship between fluency and comprehension. For example, if fluency is co- 
determined by word reading accuracy, then the correlation of fluency with comprehension may be inflated because of shared accuracy variance rather than due to a frank contribution of efficiency to comprehension. Indeed, there is some evidence that word fluency makes no unique contribution to comprehension when accuracy is accounted for (Kim, Park, \& Park, 2015; Protopapas et al., 2013; Torppa et al., 2016) or when a reliable oral language construct can take up variance shared with decoding accuracy (e.g., Foorman et al., 2015). From this perspective, a typical word reading efficiency test (such as the Test of Word Reading Efficiency; Torgesen et al., 1999) can be an excellent choice for the identification of children who have either accuracy or rate limitations, precisely because of its confounded nature.

Word reading fluency must also be distinguished from text (oral) reading fluency, which is defined by reading a meaningful passage and is, unsurprisingly, interdependent with understanding of the text (e.g., Eason et al., 2013; Jenkins et al., 2003; Kim, 2015; Kim \& Wagner, 2015). In other words, oral reading fluency tasks confound both accuracy and comprehension with the construct we advocate here, namely cascading efficiency. This makes them appropriate for the identification of children with reading difficulties (e.g., Arnesen et al., 2017; Baker et al., 2015; Morlini, Stella, \& Scorza, 2014), as differences between groups with and without dyslexia exhibit maximum effect sizes for text fluency measures, compared to other phonological, reading, or spelling measures (Protopapas \& Skaloumbakas, 2008). If, however, one is interested in the efficiency of processing word sequences, an approach to fluency that is stripped of accuracy limitations and comprehension confounds may be preferable.

In sum, we suggest that using a purer metric of word-level efficiency, such as the speed of reading aloud lists of unrelated familiar words, is informative regarding the mechanics of reading skill and relevant for our understanding and assessment of 
reading fluency. This should not be viewed as ignoring comprehension (which is, after all, the purpose of reading) but, rather, as an attempt to separate distinct dimensions of reading skill, much as researchers have long distinguished between single word recognition and reading comprehension with the goal of eventually understanding reading in its totality. Importantly, we do not advocate using a serial word reading measure as a proxy for overall reading skill, either by supplanting oral reading fluency or by prioritizing speed over comprehension. Rather, our proposal goes in the direction away from a single general measure of reading skill, highlighting dissociations among different cognitive mechanisms and sources of individual differences in reading.

\section{Caveats and Conclusion}

We have used word list reading speed to expose variance we consider crucial for word reading efficiency and oral text reading fluency, claiming that the same word-level cognitive mechanisms must be involved. We have also implicitly assumed, without offering any evidence, that it is also relevant for silent reading, where inner speech presumably replaces articulation as a rate-limiting factor. We see great advantages to this approach, because one can investigate word-level processing efficiency free from accuracy and comprehension confounds, and because oral reading provides observable time points signaling each element's processing. We believe that, in conjunction with eye tracking providing observable time points signaling the onset of engagement with each element, future studies can make headway toward understanding fluency by focusing on the distinction between single-item and multiple-item naming and reading. It remains to be seen whether the resulting theoretical progress can indeed encompass typical fluency measures and eventually extend all the way to silent reading or what other constructs may need to be recruited.

Although we have used rapid naming to expose the purported skill domain of 
cascaded processing, thanks to the well-studied relationships between RAN tasks and reading fluency, we are in no way committed to RAN being the only-or even the bestindicator of the kind of serial processing required for reading fluency. The association between RAN and fluency is merely an opportunistic observation that may help us study the cognitive mechanisms of reading fluency (see de Jong, 2011, for a related argument). RAN seems to work because of the simple and highly familiar visual-verbal associations afforded; but it is probably an imperfect measure of cascaded processing. There may be additional or alternative tasks that can help, such as tasks indexing other kinds of speeded multi-element processing (Kruk \& Luther Ruban, in press; Marx et al., 2016; Onochie-Quintanilla, Defior, \& Simpson, 2017; van den Boer, van Bergen, \& de Jong, 2015). The important question concerns the relationship between discrete and serial word processing and the additional word-level cognitive mechanisms underlying word reading fluency, without involvement of supra-lexical processing regarding sentence-level syntax and semantics.

In conclusion, our study suggests that understanding reading fluency will require an additional word-level construct, beyond the automaticity of individual word recognition, which concerns the efficiency of endogenously scheduling the processing of item sequences (termed "cascaded" processing). This seems to constitute a distinct skill domain, corresponding to the "serial naming" latent variable in our analyses, which can be indexed by rapid serial naming-and possibly other-tasks involving multi-element processing. This domain limits sequential processing efficiency once individual elements have attained sufficient automaticity to permit unmediated, single-chunk processing. Further study should determine whether this idea is on the right track to explain the full range of empirical patterns associated with the development of reading fluency, as well as its correlates and failures. 


\section{Acknowledgments}

We thank Sonia Loui, Dimitris Sagris, and Iliana Kolotoura for help administering the tasks and for processing the responses. A preliminary report of these data was presented at the 23rd Annual Conference of the Society for the Scientific Study of Reading (Porto, Portugal, 13-16 July 2016). 


\section{References}

Al Dahhan, N. Z., Kirby, J. R., \& Munoz, D. P. (2016). Understanding reading and reading difficulties through naming speed tasks: Bridging the gaps among neuroscience, cognition, and education. AERA Open, 2, 2332858416675346.

Altani, A., Georgiou, G. K., Deng, C., Cho, J.-R., Katopodi, K., Wei, W., \& Protopapas, A. (2017a) . Is processing of symbols and words influenced by writing system? Evidence from Chinese, Korean, English, and Greek. Journal of Experimental Child Psychology, 164, 117-135.

Altani, A., Protopapas, A., \& Georgiou, G. K. (2017b). The contribution of executive functions to naming digits, objects, and words. Reading and Writing, 30, 121-141.

Anastasiou, D., \& Protopapas, A. (2015). Difficulties in lexical stress versus difficulties in segmental phonology among adolescents with dyslexia. Scientific Studies of Reading, 19, 31-50.

Arnesen, A., Braeken, J., Baker, S., Meek-Hansen, W., Ogden, T., \& Melby-Lervåg, M. (2017). Growth in oral reading fluency in a semitransparent orthography: Concurrent and predictive relations with reading proficiency in Norwegian, Grades 2-5. Reading Research Quarterly, 52, 177-201.

Baker, D. L., Biancarosa, G., Park, B. J., Bousselot, T., Smith, J. L., Baker, S. K., ... \& Tindal, G. (2015). Validity of CBM measures of oral reading fluency and reading comprehension on high-stakes reading assessments in Grades 7 and 8. Reading and Writing, 28, 57-104.

Bowey, J. A., McGuigan, M., \& Ruschena, A. (2005). On the association between serial naming speed for letters and digits and word reading skill: Towards a developmental account. Journal of Research in Reading, 28, 400-422.

Bowey, J. A., Storey, T., \& Ferguson, A. N. (2004). The association between continuous 
naming speed and word reading skills in fourth- to sixth-grade children. Australian Journal of Psychology, 56, 155-163.

Breznitz, Z. (2006). Fluency in reading: Synchronization of processes. Mahwah, NJ: Erlbaum.

Buswell, G. T. (1921). The relationship between eye-perception and voice-response in reading. Journal of Educational Psychology, 12, 217-227.

Carver, R. P. (1997). Reading for one second, one minute, or one year from the perspective of rauding theory. Scientific Studies of Reading, 1, 3-43.

Cummings, K. D., \& Petscher, Y. (Eds.) (2016). The fluency construct: Curriculum-based measurement concepts and applications. New York: Springer.

de Jong, P. F. (2011). What discrete and serial rapid automatized naming can reveal about reading. Scientific Studies of Reading, 15, 314-337.

De Luca, M., Pontillo, M., Primativo, S., Spinelli, D., \& Zoccolotti, P. (2013). The eye-voice lead during oral reading in developmental dyslexia. Frontiers in Human Neuroscience, 7, 696.

Eason, S. H., Sabatini, J., Goldberg, L., Bruce, K., \& Cutting, L. E. (2013). Examining the relationship between word reading efficiency and oral reading rate in predicting comprehension among different types of readers. Scientific Studies of Reading, $17,199-223$.

Ehri, L. C. (2005). Learning to read words: Theory, findings, and issues. Scientific Studies of Reading, 9, 167-188.

Ehri, L. C. (2014). Orthographic mapping in the acquisition of sight word reading, spelling memory, and vocabulary learning. Scientific Studies of Reading, 18, 5-21.

Foorman, B. R., Koon, S., Petscher, Y., Mitchell, A., \& Truckenmiller, A. (2015). Examining general and specific factors in the dimensionality of oral language and reading in 
4th-10th grades. Journal of Educational Psychology, 107, 884-899.

Fuchs, L. S., Fuchs, D., Hosp, M. K., \& Jenkins, J. R. (2001). Oral reading fluency as an indicator of reading competence: A theoretical, empirical, and historical analysis. Scientific Studies of Reading, 5, 239-256.

Gasperini, F., Brizzolara, D., Cristofani, P., Casalini, C., \& Chilosi, A. M. (2014). The contribution of discrete-trial naming and visual recognition to rapid automatized naming deficits of dyslexic children with and without a history of language delay. Frontiers in Human Neuroscience, 8, 652.

Georgiou, G., Papadopoulos, T. C., \& Kaizer, E. L. (2014). Different RAN components predict reading at different points in time. Reading and Writing: An Interdisciplinary Journal, 27, 1379-1394.

Georgiou, G., Parrila, R., \& Kirby, J. (2009). RAN components and reading development from Grade 3 to Grade 5: What underlies their relationship? Scientific Studies of Reading, 13, 508-534.

Gordon, P. C., \& Hoedemaker, R. S. (2016). Effective scheduling of looking and talking during rapid automatized naming. Journal of Experimental Psychology: Human Perception and Performance, 42, 742-760.

Heikkilä, R., Aro, M., Närhi, V., Westerholm, J., \& Ahonen, T. (2013). Does training in syllable recognition improve reading speed? A computer-based trial with poor readers from second and third grade. Scientific Studies of Reading, 17, 398-414.

Hothorn, T., Bretz, F., \& Westfall, P. (2008). Simultaneous inference in general parametric models. Biometrical Journal, 50, 346-363.

Hudson, R. F., Pullen, P. C., Lane, H. B., \& Torgesen, J. K. (2009). The complex nature of reading fluency: A multidimensional view. Reading \& Writing Quarterly, 25, 4-32.

Hudson, R. F., Torgesen, J. K., Lane, H. B., \& Turner, S. J. (2012). Relations among reading 
skills and sub-skills and text-level reading proficiency in developing readers. Reading and Writing, 25, 483-507.

Jenkins, J. R., Fuchs, L. S., Van Den Broek, P., Espin, C., \& Deno, S. L. (2003). Sources of individual differences in reading comprehension and reading fluency. Journal of Educational Psychology, 95, 719-729.

Kim, Y. S. G. (2015). Developmental, component-based model of reading fluency: An investigation of predictors of word-reading fluency, text-reading fluency, and reading comprehension. Reading Research Quarterly, 50, 459-481.

Kim, Y. S. G., Park, C., \& Park, Y. (2015). Dimensions of discourse level oral language skills and their relation to reading comprehension and written composition: an exploratory study. Reading and Writing, 28, 633-654.

Kim, Y. S. G., \& Wagner, R. K. (2015). Text (oral) reading fluency as a construct in reading development: An investigation of its mediating role for children from Grades 1 to 4. Scientific Studies of Reading, 19, 224-242.

Kruk, R. S., \& Luther Ruban, C. (in press). Beyond phonology: Visual processes predict alphanumeric and nonalphanumeric rapid naming in poor early readers. Journal of Learning Disabilities, doi:10.1177/0022219416678406.

Kuhn, M. R., Schwanenflugel, P. J., \& Meisinger, E. B. (2010). Aligning theory and assessment of reading fluency: Automaticity, prosody, and definitions of fluency. Reading Research Quarterly, 45, 230-251.

Kuhn, M. R., \& Stahl, S. A. (2003). Fluency: A review of developmental and remedial practices. Journal of Educational Psychology, 95, 3-21.

LaBerge, D., \& Samuels, S. J. (1974). Toward a theory of automatic information processing in reading. Cognitive Psychology, 6, 293-323.

Landerl, K., \& Wimmer, H. (2008). Development of word reading fluency and spelling in 
a consistent orthography: An 8-year follow-up. Journal of Educational Psychology, $100,150-161$.

Landerl, K., Wimmer, H., \& Frith, U. (1997). The impact of orthographic consistency on dyslexia: A German-English comparison. Cognition, 63, 315-334.

Laubrock, J., \& Kliegl, R. (2015). The eye-voice span during reading aloud. Frontiers in Psychology, 6, 1432.

Lee, J., \& Yoon, S. Y. (2017). The effects of repeated reading on reading fluency for students with reading disabilities: A meta-analysis. Journal of Learning Disabilities, 50, 213-224.

Liu, C.-N., \& Georgiou, G. (2017). Cognitive and environmental correlates of rapid automatized naming in Chinese kindergarten children. Journal of Educational Psychology, 109, 465-476.

Marx, C., Hutzler, F., Schuster, S., \& Hawelka, S. (2016). On the development of parafoveal preprocessing: Evidence from the incremental boundary paradigm. Frontiers in Psychology, 7, 514.

Morlini, I., Stella, G., \& Scorza, M. (2014). A new procedure to measure children's reading speed and accuracy in Italian. Dyslexia, 20, 54-73.

National Reading Panel (2000). Report of the national reading panel: Teaching children to read, Reports of the subgroups. NIH Pub. No. 00-4754. U.S. Department of Health and Human Services.

Neale, M. C., Hunter, M. D., Pritikin, J. N., Zahery, M., Brick, T. R., Kirkpatrick, R. M., Estabrook, R., et al. (2016). OpenMX 2.0: Extended structural equation and statistical modeling. Psychometrica, 81, 535-549.

Onochie-Quintanilla, E., Defior, S., \& Simpson, I. C. (2017). Visual multi-element processing as a pre-reading predictor of decoding skill. Journal of Memory and 
Language, 94, 134-148.

Pan, J., Yan, M., Laubrock, J., Shu, H., \& Kliegl, R. (2013). Eye-voice span during rapid automatized naming of digits and dice in Chinese normal and dyslexic children. Developmental Science, 16, 967-979.

Pinheiro, J., Bates, D., DebRoy, S., Sarkar, D., \& R Core Team (2016). nlme: Linear and nonlinear mixed effects models. cran.r-project.org/package=nlme

Poulsen, M., \& Elbro, C. (2013). What's in a name depends on the type of name: The relationship between semantic and phonological access, reading fluency, and reading comprehension. Scientific Studies of Reading, 17, 303-314.

Protopapas, A. (2007). Check Vocal: A program to facilitate checking the accuracy and response time of vocal responses from DMDX. Behavior Research Methods, 39, 859-862.

Protopapas, A., Altani, A., \& Georgiou, G. K. (2013). Development of serial processing in reading and rapid naming. Journal of Experimental Child Psychology, 116, 914929.

Protopapas, A., Markatou, A., Samaras, E., \& Piokos, A. (2017). Shape and color naming are inherently asymmetrical: Evidence from practice-based interference. Cognition, 158, 122-133.

Protopapas, A., Mouzaki, A., Sideridis, G. D., Kotsolakou, A., \& Simos, P. G. (2013). The role of vocabulary in the context of the simple view of reading. Reading \& Writing Quarterly, 29, 168-202.

Protopapas, A., \& Skaloumbakas, C. (2007). Traditional and computer-based screening and diagnosis of reading disabilities in Greek. Journal of Learning Disabilities, 40, $15-36$.

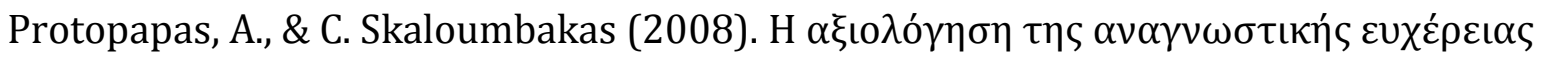




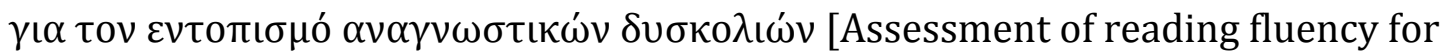
the identification of reading difficulties]. Psychologia, 15, 267-289.

Protopapas, A., Skaloumbakas, C., \& Bali, P. (2008). Validation of unsupervised computer-based screening for reading disability in the Greek elementary Grades 3 and 4. Learning Disabilities: A Contemporary Journal, 6, 45-69.

Protopapas, A., \& Vlahou, E. L. (2009). A comparative quantitative analysis of Greek orthographic transparency. Behavior Research Methods, 41, 991-1008.

Protopapas, A., Tzakosta, M., Chalamandaris, A., \& Tsiakoulis, P. (2012). IPLR: An online resource for Greek word-level and sublexical information. Language Resources \& Evaluation, 46, 449-459.

R development core team. (2016). R: A language and environment for statistical computing. Vienna: R Foundation for Statistical Computing (www.R-project.org).

Rasinski, T. V., Reutzel, D. R., Chard, D., \& Linan-Thompson, S. (2012). Reading fluency. In M. L. Kamil, P. D. Pearson, E. Birr Moje, \& P. Afflerbach (Eds.), Handbook of reading research, Vol. IV (pp. 286-319). New York: Routledge.

Rasinski, T., Rikli, A., \& Johnston, S. (2009). Reading fluency: More than automaticity? More than a concern for the primary grades? Literacy Research and Instruction, $48,350-361$.

Roelofs, A. (2003). Goal-referenced selection of verbal action: Modeling attentional control in the Stroop task. Psychological Review, 110, 88-125.

Roelofs, A. (2006). Functional architecture of naming dice, digits, and number words. Language and Cognitive Processes, 21, 78-111.

Samuels, S. J. (2006). Toward a model of reading fluency. In S. J. Samuels \& A. E. Farstrup (Eds.), What research has to say about fluency instruction (pp. 24-46). Newark, DE: International Reading Association. 
Schwanenflugel, P. J., Hamilton, A. M., Kuhn, M. R., Wisenbaker, J. M., \& Stahl, S. A. (2004). Becoming a fluent reader: reading skill and prosodic features in the oral reading of young readers. Journal of Educational Psychology, 96, 119-129.

Share, D. L. (2008). On the anglocentricities of current reading research and practice: The perils of overreliance on an "outlier" orthography. Psychological Bulletin, $134,584-615$.

Silva, S., Reis, A., Casaca, L., Petersson, K. M., \& Faísca, L. (2016). When the eyes no longer lead: Familiarity and length effects on eye-voice span. Frontiers in Psychology, 7, 1720.

Torgesen, J. K. (2005). Recent discoveries on remedial instructions for children with dyslexia. In M. J. Snowling \& C. Hulme (Eds.), The science of reading: A handbook (pp. 521-537). Malden, MA: Blackwell.

Torgesen, J. K., Wagner, R. K., \& Rashotte, C. A. (1999). Test of word reading efficiency. Austin, TX: Pro-Ed.

Torppa, M., Georgiou, G., Lerkkanen, M.-K., Niemi, P., Poikkeus, A.-M., \& Nurmi, J.-E. (2016). Examining the "simple view of reading" in a transparent orthography: A longitudinal study from Kindergarten to Grade 3. Merrill Palmer Quarterly, 62, 179-206.

van den Boer, M., \& de Jong, P. F. (2015). Parallel and serial reading processes in children's word and nonword reading. Journal of Educational Psychology, 107, 141-151.

van den Boer, M., Georgiou, G. K., \& de Jong, P. F. (2016). Naming of short words is (almost) the same as naming of alphanumeric symbols: Evidence from two orthographies. Journal of Experimental Child Psychology, 144, 152-165.

van den Boer, M., van Bergen, E., \& de Jong, P. F. (2015). The specific relation of visual 
attention span with reading and spelling in Dutch. Learning and Individual Differences, 39, 141-149.

van den Bos, K. P., Zijlstra, B. J. H., \& van den Broeck, W. (2003). Specific relations between alphanumeric-naming speed and reading speeds of monosyllabic and multisyllabic words. Applied Psycholinguistics, 24, 407-430.

Van Heuven, W. J. B., Mandera, P., Keuleers, E., \& Brysbaert, M. (2014). Subtlex-UK: A new and improved word frequency database for British English. Quarterly Journal of Experimental Psychology, 67, 1176-1190.

Wolf, M., \& Bowers, P. (1999). The "double-deficit hypothesis" for the developmental dyslexias. Journal of Educational Psychology, 91, 1-24.

Wolf, M., Bowers, P. G., \& Biddle, K. (2000). Naming-speed processes, timing, and reading: A conceptual review. Journal of Learning Disabilities, 33, 387-407.

Wolf, M., \& Katzir-Cohen, T. (2001). Reading fluency and its intervention. Scientific Studies of Reading, 5, 211-239.

Zoccolotti, P., De Luca, M., Lami, L., Pizzoli, C., Pontillo, M., \& Spinelli, D. (2013). Multiple stimulus presentation yields larger deficits in children with developmental dyslexia: a study with reading and RAN-type tasks. Child Neuropsychology, 19, $639-647$.

Zoccolotti, P., De Luca, M., Marinelli, C. V., \& Spinelli, D. (2014). Modeling individual differences in text reading fluency: a different pattern of predictors for typically developing and dyslexic readers. Frontiers in Psychology, 5, 1374.

Zoccolotti, P., De Luca, M., \& Spinelli, D. (2015). Discrete versus multiple word displays: A re-analysis of studies comparing dyslexic and typically developing children. Frontiers in Psychology, 6, 1530. 


\section{Appendix}

Digits: 2, 3, 5, 6

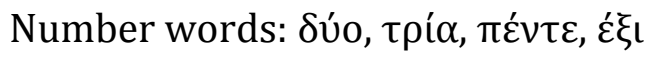

Object words: $\mu \pi \alpha \dot{\alpha} \lambda \alpha$, ó $\pi \lambda o, \pi \lambda o$ ó, $\omega \rho \alpha$

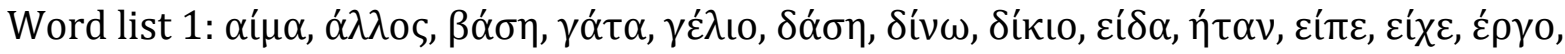

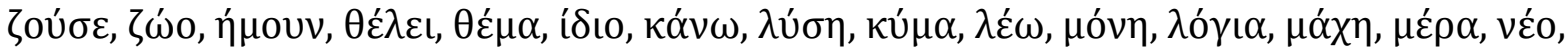

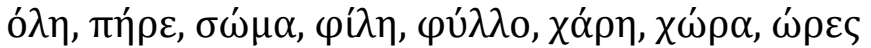

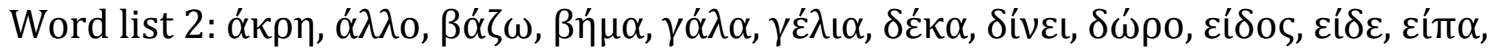

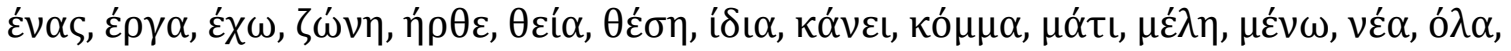

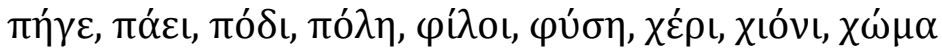

\begin{tabular}{|c|c|c|c|c|}
\hline & $M$ & $S D$ & $\min$ & $\max$ \\
\hline \multicolumn{5}{|l|}{ Number words } \\
\hline Number of letters & 3.8 & 1.0 & 3 & 5 \\
\hline Number of phonemes & 3.8 & 0.5 & 3 & 4 \\
\hline Number of syllables & 2.0 & - & 2 & 2 \\
\hline Printed frequency (children) & 5.5 & 0.4 & 5.1 & 6.0 \\
\hline Printed frequency (adult) & 5.6 & 0.5 & 5.2 & 6.3 \\
\hline \multicolumn{5}{|l|}{ Object words } \\
\hline Number of letters & 4.2 & 1.0 & 3 & 5 \\
\hline Number of phonemes & 3.8 & 0.5 & 3 & 4 \\
\hline Number of syllables & 2.0 & - & 2 & 2 \\
\hline Printed frequency (children) & 5.1 & 0.6 & 4.5 & 5.9 \\
\hline Printed frequency (adult) & 5.0 & 0.5 & 4.6 & 5.6 \\
\hline \multicolumn{5}{|l|}{ Word list 1} \\
\hline Number of letters & 4.1 & 0.6 & 3 & 5 \\
\hline Number of phonemes & 3.8 & 0.4 & 3 & 4 \\
\hline Number of syllables & 2.0 & - & 2 & 2 \\
\hline Printed frequency (children) & 5.2 & 0.5 & 4.6 & 6.5 \\
\hline Printed frequency (adult) & 5.1 & 0.7 & 3.9 & 6.5 \\
\hline \multicolumn{5}{|l|}{ Word list 2} \\
\hline Number of letters & 4.1 & 0.5 & 3 & 5 \\
\hline Number of phonemes & 3.8 & 0.4 & 3 & 4 \\
\hline Number of syllables & 2.0 & - & 2 & 2 \\
\hline Printed frequency (children) & 5.3 & 0.4 & 4.4 & 6.1 \\
\hline Printed frequency (adult) & 5.0 & 0.6 & 3.7 & 6.0 \\
\hline
\end{tabular}

Note. Printed word frequencies are in the Zipf scale (Van Heuven et al., 2014). Children's frequencies are based on the language arts textbooks for Grades 1-6; adult frequencies are from the IPLR C corpus (Protopapas et al., 2012). 
Table 1

Descriptive statistics for each grade and task

\begin{tabular}{|c|c|c|c|c|c|c|c|c|c|c|c|c|c|c|c|}
\hline & \multicolumn{5}{|c|}{ Grade 1} & \multicolumn{5}{|c|}{ Grade 3} & \multicolumn{5}{|c|}{ Grade 5} \\
\hline & $N$ & $M$ & $S D$ & Skew & Kurt & $N$ & $M$ & $S D$ & Skew & Kurt & $N$ & $M$ & $S D$ & Skew & Kurt \\
\hline \multicolumn{16}{|l|}{ Serial tasks } \\
\hline Digits & 100 & 1.38 & 0.27 & 0.04 & -0.43 & 103 & 1.91 & 0.34 & -0.17 & -0.47 & 99 & 2.13 & 0.38 & -0.59 & 0.47 \\
\hline Number words & 100 & 1.24 & 0.31 & -0.02 & 0.27 & 103 & 1.92 & 0.33 & -0.33 & -0.08 & 99 & 2.16 & 0.38 & -0.33 & 0.06 \\
\hline Words & 99 & 0.67 & 0.26 & 0.54 & -0.36 & 103 & 1.40 & 0.40 & -0.01 & 0.02 & 98 & 1.67 & 0.35 & -0.14 & -0.49 \\
\hline Dice & 100 & 1.12 & 0.27 & 0.00 & -0.17 & 103 & 1.55 & 0.30 & 0.01 & -0.35 & 99 & 1.72 & 0.34 & 0.24 & 0.25 \\
\hline Objects & 100 & 0.74 & 0.16 & 0.34 & -0.64 & 103 & 1.05 & 0.20 & 0.33 & -0.35 & 99 & 1.20 & 0.23 & 0.19 & 0.77 \\
\hline \multicolumn{16}{|c|}{ Discrete tasks } \\
\hline Digits & 100 & 0.90 & 0.15 & 0.29 & 0.18 & 103 & 1.13 & 0.16 & 0.39 & 0.95 & 99 & 1.21 & 0.17 & 0.23 & -0.10 \\
\hline Number words & 100 & 0.86 & 0.15 & 0.08 & -0.23 & 103 & 1.14 & 0.17 & 0.31 & 0.78 & 99 & 1.20 & 0.18 & 0.15 & -0.44 \\
\hline Words & 98 & 0.60 & 0.14 & 0.04 & -0.57 & 103 & 0.96 & 0.17 & -0.04 & 0.16 & 99 & 1.06 & 0.16 & 0.00 & -0.50 \\
\hline Dice & 100 & 0.80 & 0.15 & -0.03 & -0.02 & 103 & 1.00 & 0.16 & 0.33 & 0.10 & 98 & 1.06 & 0.15 & 0.40 & 0.07 \\
\hline Objects & 100 & 0.69 & 0.10 & 0.53 & 0.43 & 103 & 0.89 & 0.13 & 0.28 & 0.45 & 99 & 0.94 & 0.13 & 0.21 & -0.10 \\
\hline
\end{tabular}

Note: Skew = skewness; Kurt = kurtosis. 
Table 2

Intercorrelations among tasks for each grade

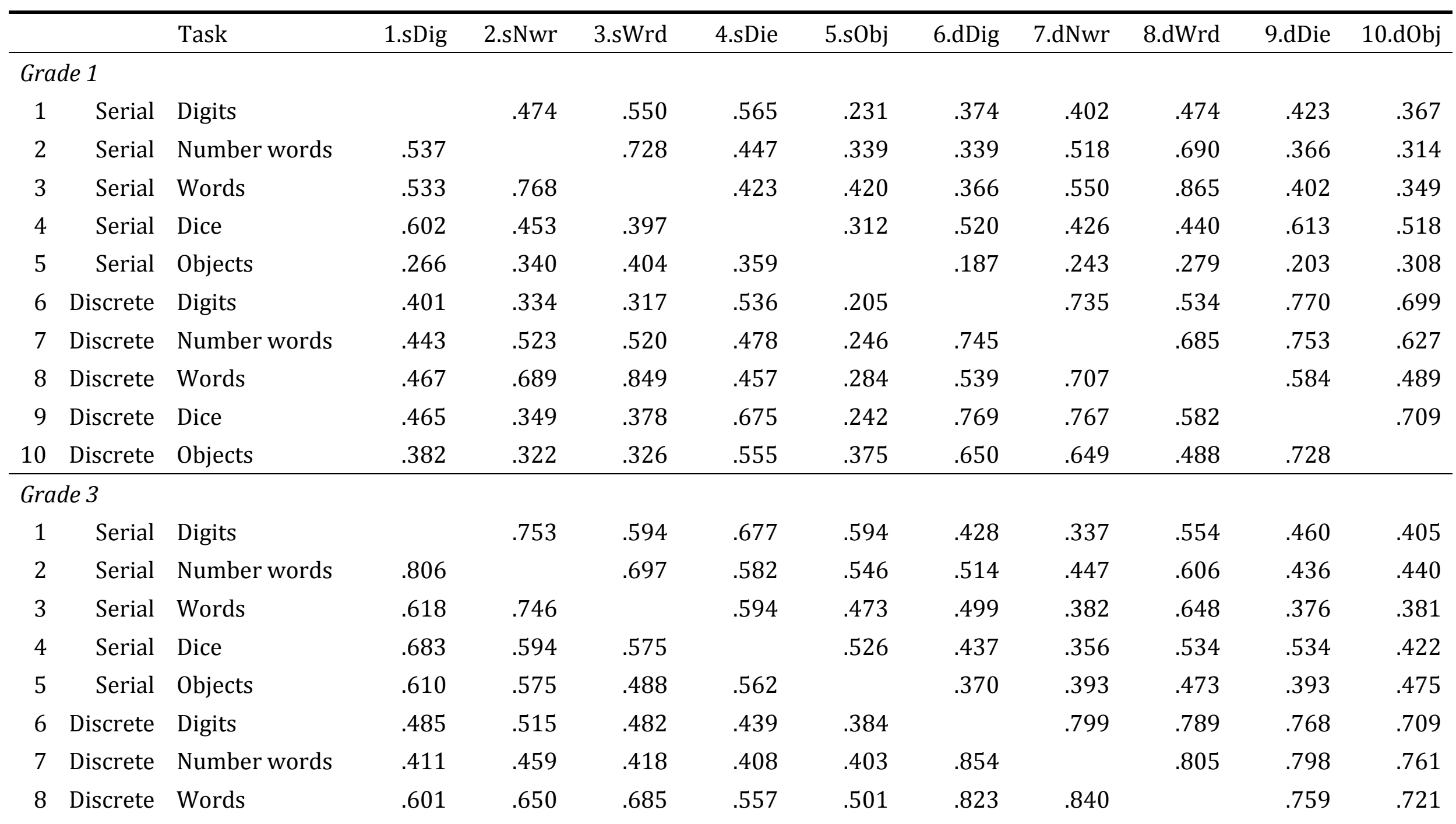




\begin{tabular}{|c|c|c|c|c|c|c|c|c|c|c|c|c|}
\hline 9 & Discrete & Dice & .504 & .453 & .383 & .525 & .380 & .830 & .845 & .777 & & .758 \\
\hline 10 & Discrete & Objects & .468 & .477 & .406 & .437 & .488 & .797 & .816 & .769 & .799 & \\
\hline \multicolumn{13}{|c|}{ Grade 5} \\
\hline 2 & Serial & Number words & .820 & & .758 & .473 & .415 & .370 & .480 & .514 & .422 & .472 \\
\hline 3 & Serial & Words & .655 & .769 & & .481 & .480 & .411 & .533 & .543 & .403 & .459 \\
\hline 6 & Discrete & Digits & .415 & .421 & .419 & .262 & .354 & & .854 & .778 & .765 & .695 \\
\hline 7 & Discrete & Number words & .488 & .510 & .537 & .355 & .414 & .861 & & .825 & .771 & .730 \\
\hline 8 & Discrete & Words & .454 & .515 & .564 & .208 & .312 & .777 & .832 & & .674 & .658 \\
\hline 9 & Discrete & Dice & .539 & .481 & .410 & .511 & .511 & .771 & .769 & .677 & & .722 \\
\hline
\end{tabular}

Note: For each grade, Spearman's $\rho$ above the diagonal; Pearson's $r$ below the diagonal. $\mathrm{s}=$ serial, $\mathrm{d}=$ discrete, Dig $=$ digits, Nwr $=$ number words, Wrd $=$ words, Die $=$ dice, Obj $=$ objects. 
Table 3

Correlation (Pearson's r) of serial words to discrete words and to serial and discrete digits by grade

\begin{tabular}{ccccc}
\hline Grade & $\begin{array}{c}\text { Serial words }- \\
\text { Discrete words }\end{array}$ & $\begin{array}{c}\text { Serial words }- \\
\text { Serial digits }\end{array}$ & $\begin{array}{c}\text { Serial words }- \\
\text { Discrete digits }\end{array}$ & $\begin{array}{c}\text { Discrete words - } \\
\text { Discrete digits }\end{array}$ \\
\hline 1 & .85 & .53 & .32 & .54 \\
$2^{\mathrm{a}}$ & .83 & .52 & .37 & .50 \\
3 & .69 & .62 & .48 & .82 \\
5 & .56 & .66 & .42 & .78 \\
$6^{\mathrm{a}}$ & .42 & .61 & .26 & .68 \\
\hline
\end{tabular}

a Data for Grades $2(N=102)$ and $6(N=105)$ are from Protopapas et al. (2013). 
Table 4

Post-hoc linear contrast results for the interactions between grade and format (serial vs. discrete)

\begin{tabular}{llrrr}
\hline Grades & Task & Est. & $z$ & $p$ \\
\hline G1 vs. G3 & Digits & 0.310 & 8.839 & $<.001$ \\
G1 vs. G3 & Number words & 0.411 & 11.709 & $<.001$ \\
G1 vs. G3 & Words & 0.380 & 10.762 & $<.001$ \\
G1 vs. G3 & Dice & 0.074 & 2.101 & .287 \\
G1 vs. G3 & Objects & 0.121 & 3.437 & .006 \\
\hline G3 vs. G5 & Digits & 0.130 & 3.694 & .002 \\
G3 vs. G5 & Number words & 0.173 & 4.909 & $<.001$ \\
G3 vs. G5 & Words & 0.161 & 4.563 & $<.001$ \\
G3 vs. G5 & Dice & 0.104 & 2.956 & .030 \\
G3 vs. G5 & Objects & 0.096 & 2.727 & .060 \\
\hline
\end{tabular}

Note: $\mathrm{G}=$ Grade; Est. = contrast estimate; $p$ values are adjusted for multiple comparisons using the "single-step" method (based on the joint normal or $t$ distribution of the linear function). 


\section{Figure Captions}

Figure 1. Mean performance (items per second) for each task in each grade.

Figure 2. Constrained multi-group CFA models for serial tasks (top; $\chi^{2}=33.98, d f=23, p$ $=.066, \mathrm{NNFI}=.98, \mathrm{CFI}=.99, \mathrm{RMSEA}=.04[95 \% \mathrm{CI} .00-.07])$ and discrete tasks (bottom; $\left.\chi^{2}=27.81, d f=28, p=.475, \mathrm{NNFI}=1.00, \mathrm{CFI}=1.00, \mathrm{RMSEA}<.001[95 \% \mathrm{CI} .00-.05]\right)$. Equal signs indicate parameters constrained to be equal to those for Grade 3. All parameters are standardized. Estimates of observed means are not shown. 

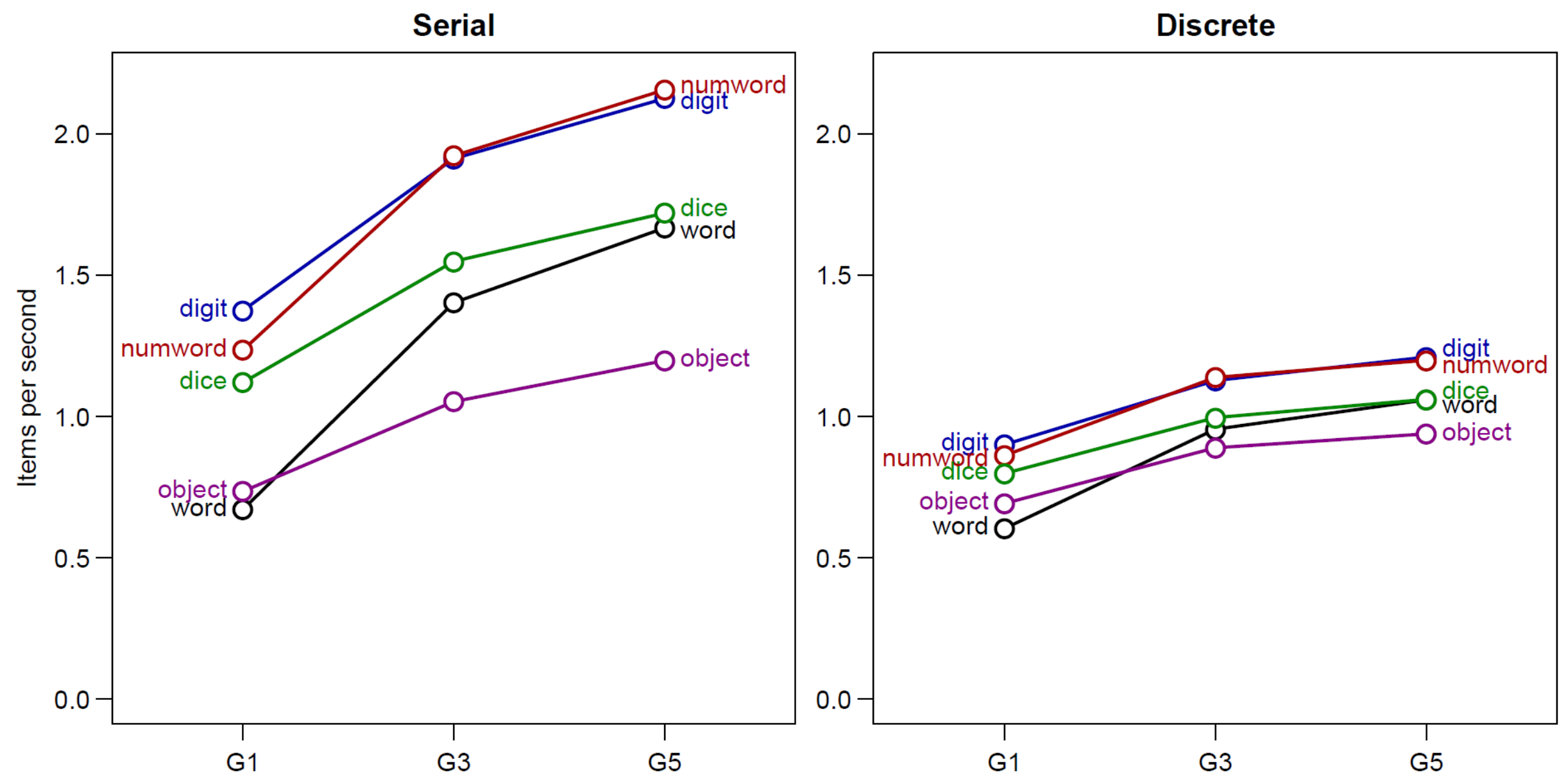
Grade 1
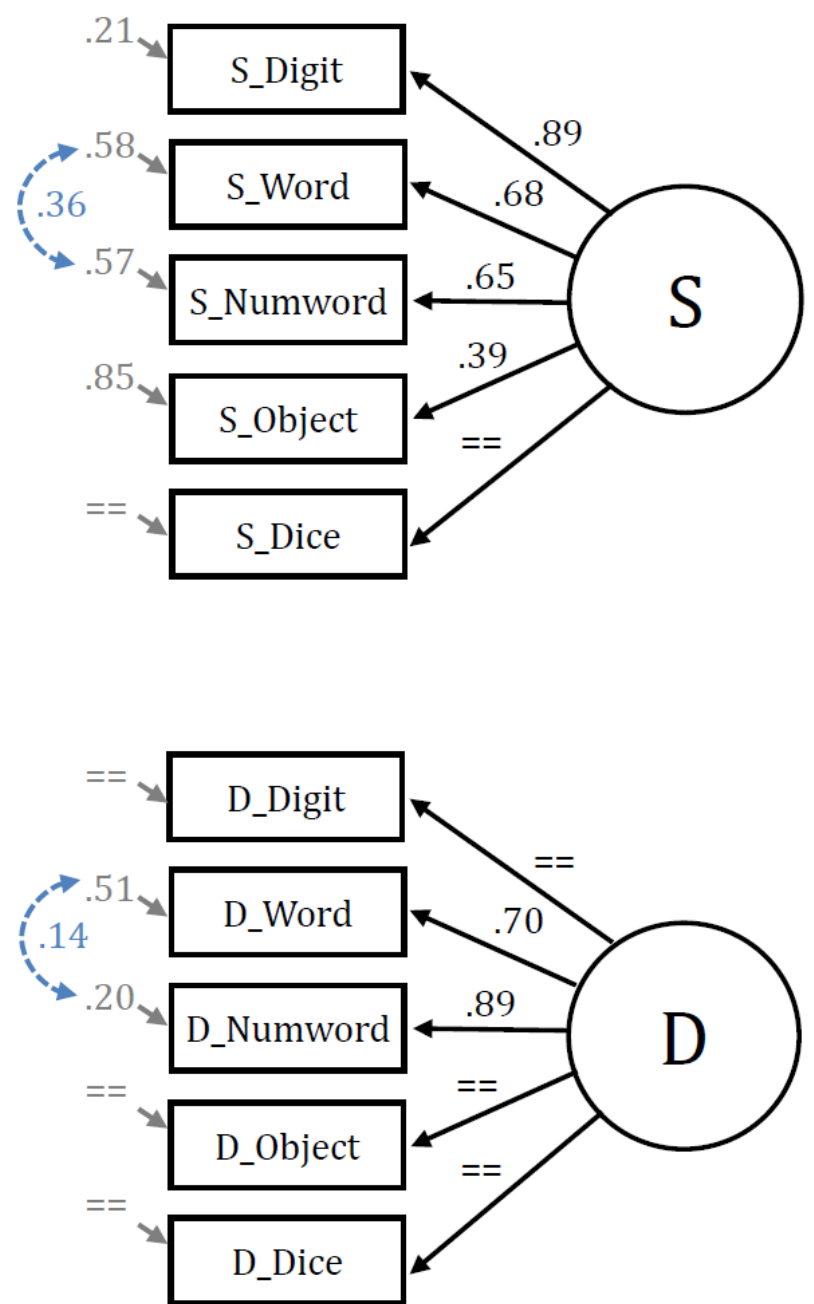

Grade 3
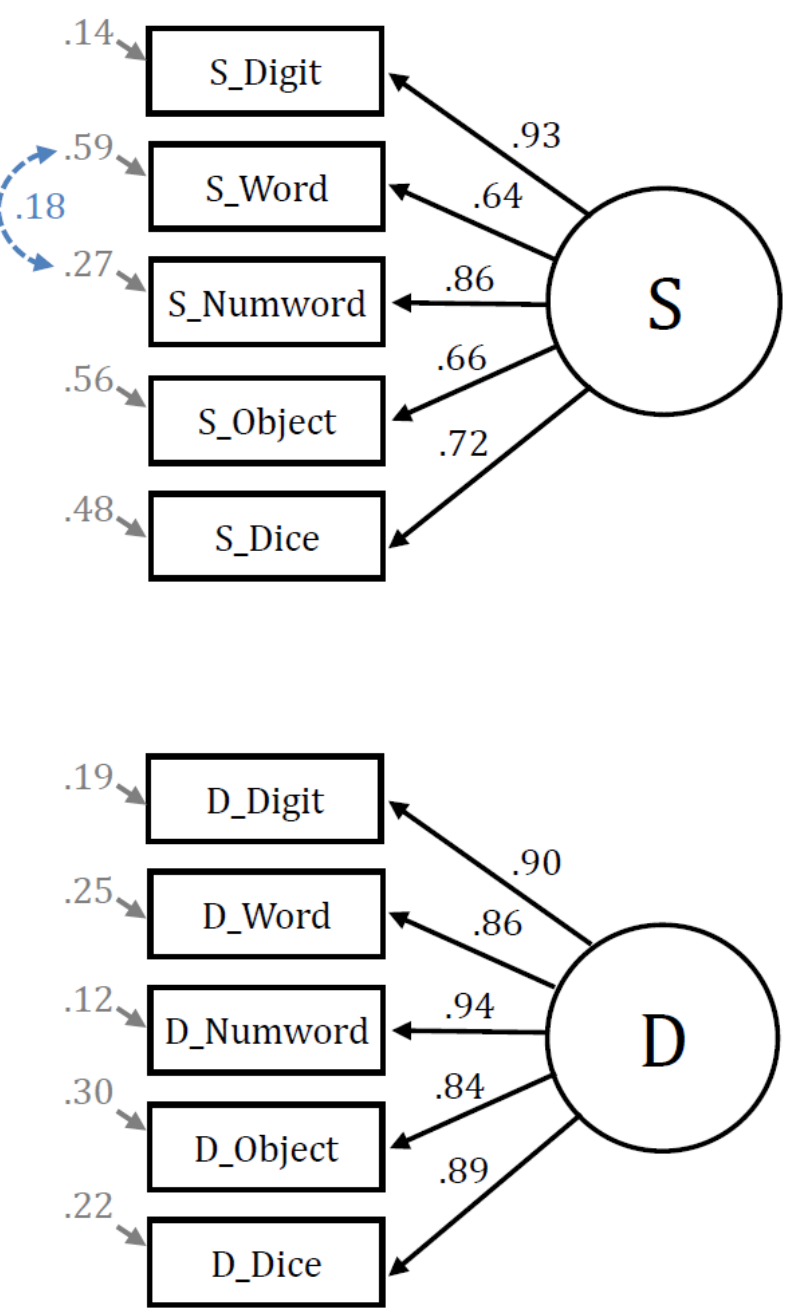

Grade 5
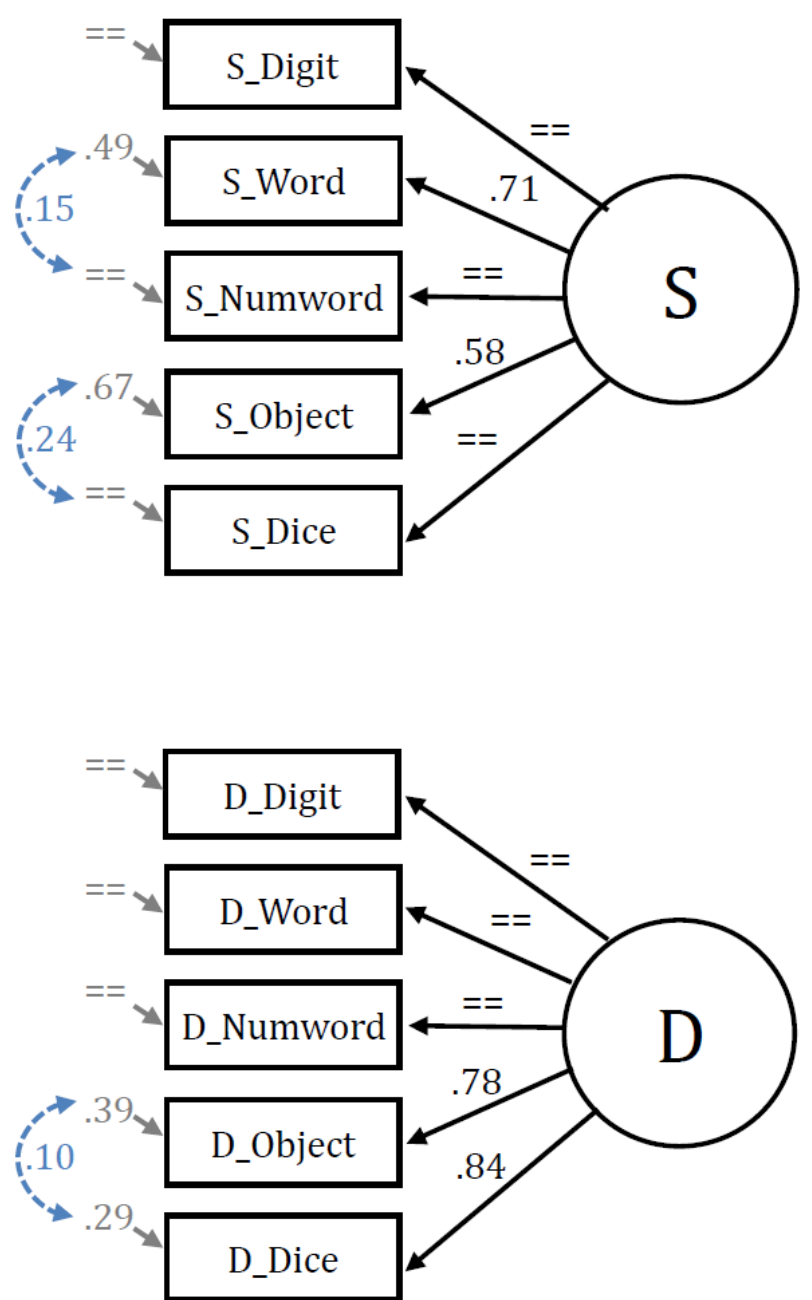